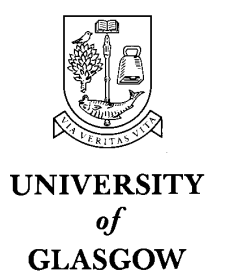

Department of Physics \& Astronomy

Experimental Particle Physics Group

Kelvin Building, University of Glasgow,

Glasgow, G12 8QQ, Scotland

GLASGOW
GLAS-PPE/1999-09

July 1999

\title{
Prompt photon processes in photoproduction at HERA
}

\author{
Sung Won Lee \\ On behalf of the ZEUS Collaboration \\ Talk given at Photon99 Conference, Freiburg, Germany \\ Slides are also available from \\ http://zedy00.desy.de/\%7Eslee/report/pp99/index.html
}

\begin{abstract}
We present results for the photoproduction of inclusive prompt photons and for prompt photons accompanied by jets, measured with the ZEUS detector at HERA. Cross sections as a function of pseudorapidity and transverse energy are presented for $5<E_{T}^{\gamma}<10 \mathrm{GeV}, E_{T}^{\text {jet }}>5 \mathrm{GeV}$ in the centre of mass energy range 120-270 GeV. Comparisons are made with predictions from leading logarithm parton shower Monte Carlos and next-to-leading order QCD calculations using currently available models of the photon structure. NLO QCD calculations describe the shape and magnitude of the measurements reasonably well.
\end{abstract}


(a)

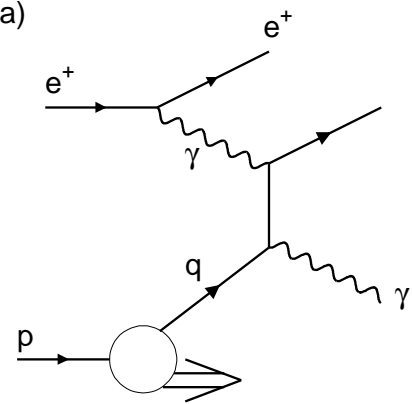

(b)

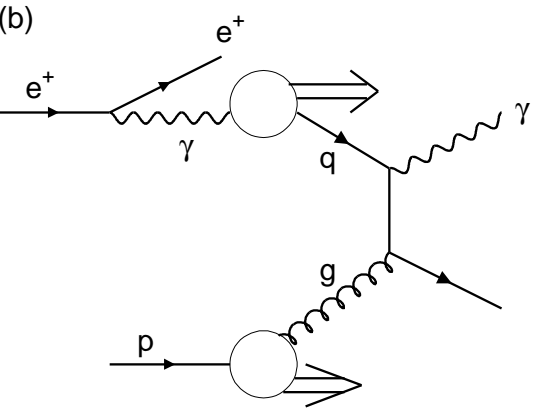

Figure 1: Example of (a) direct (pointlike) (b) resolved (hadronic) processes in LO hard photoproduction producing an outgoing prompt photon.

\section{Introduction}

Isolated high transverse energy ("prompt") photon processes at HERA (fig. 1) could yield information about the quark and gluon content of the photon, together with the gluon structure of the proton [1]. The particular virtue of prompt photon processes is that the observed final state photon emerges directly from a QCD diagram without the subsequent hadronisation which complicates the study of high $E_{T}$ quarks and gluons.

The ZEUS collaboration has recently published the first observation at HERA of prompt photons at high transverse momentum in photoproduction reactions [2], based on an integrated luminosity of $6.4 \mathrm{pb}^{-1}$. An NLO calculation by Gordon [3] was found to be in agreement with the ZEUS results, and indicates the feasibility of distinguishing between different models of the photon structure.

In the present study we extend our earlier study of prompt photon production from a data sample of 37 $\mathrm{pb}^{-1}$. Differential cross sections are given for the final state containing a prompt photon, and a prompt photon accompanying jet as a function of pseudorapidity and of transverse photon energy.

Comparison is made with several LO and NLO (next to leading order) predictions, with the goal of testing different proposed hadronic structures of the incoming photon.

\section{Event Selection}

The data used here were obtained from $e^{+} p$ running in 1996-97 at HERA, with $E_{e}=27.5 \mathrm{GeV}, E_{p}=820 \mathrm{GeV}$. The ZEUS experiment is described elsewhere 国. The major components used in the analysis are the central tracking detector (CTD) and the uranium-scintillator calorimeter(UCAL). Prompt photons are detected in the barrel section of the calorimeter (BCAL), which consists of an electromagnetic section (BEMC) followed by two hadronic sections; the BEMC consists of pointing cells of $\approx 20 \mathrm{~cm}$ length and $\approx 5 \mathrm{~cm}$ width at a minimum radius $1.23 \mathrm{~m}$ from the beamline. This width is not small enough to resolve the photons from the processes $\pi^{0} \rightarrow 2 \gamma, \eta \rightarrow 2 \gamma$ and $\eta \rightarrow 3 \pi^{0}$ on an event by event basis. It does, however, enable a partial discrimination between single photon signals and the decay product of neutral mesons.

A standard ZEUS electron finding algorithm was used to identify candidate photon signals in BCAL with measured $E_{T}^{\gamma}>4.5 \mathrm{GeV}$. The Energy loss in dead material to the measured photon energy has been corrected using MC generated single photons. This correction amounted typically to 200-300 MeV. After the photon energy correction the events were retained for final analysis if a photon candidates with transverse energy $E_{T}^{\gamma}>5 \mathrm{GeV}$ was found in the BCAL. To identify jets, a cone jet finding algorithm [5] was used. Jet with $E_{T}^{\text {jet }}>4.5 \mathrm{GeV}$ and pseudorapidity $-1.5<\eta^{\text {jet }}<1.8$ were accepted with a cone radius of 1 radian, where pseudorapidity is defined as $\eta=-\ln (\tan \theta / 2)$. Events with an identified DIS positron were removed, restricting the acceptance of the present analysis to incoming photons of virtuality $Q^{2} \leq 1 \mathrm{GeV}^{2}$. The quantity $y_{J B}$, defined as the sum of $\left(E-p_{Z}\right)$ over all the UCAL cells divided by twice the positron beam energy $E_{e}$, provides a measure of the fractional energy $E_{\gamma 0} / E_{e}$ of the interacting quasi-real photon. A requirement of $0.15<y_{J B}<0.7$ was imposed, the lower cut removing some residual proton-gas backgrounds and the upper cut removing remaining DIS events. Wide-angle Compton scatters were also excluded by this cut.

A photon candidate was rejected if a CTD track pointed within $0.3 \mathrm{rad}$ of it. An isolation cone was also imposed around photon candidates: within a cone of unit radius in $(\eta, \phi)$, the total $E_{T}$ from other particles was required not to exceed $0.1 E_{T}(\gamma)$. This greatly reduces backgrounds from dijet events with part of one jet misidentified as a single photon $\left(\pi^{0}, \eta\right.$, etc). In addition, as discussed in 11], it removes most dijet events in which a high $E_{T}$ photon radiating from a final state quark. A remainder of such events is included as part of the signal in the data and the theoretical calculations. 


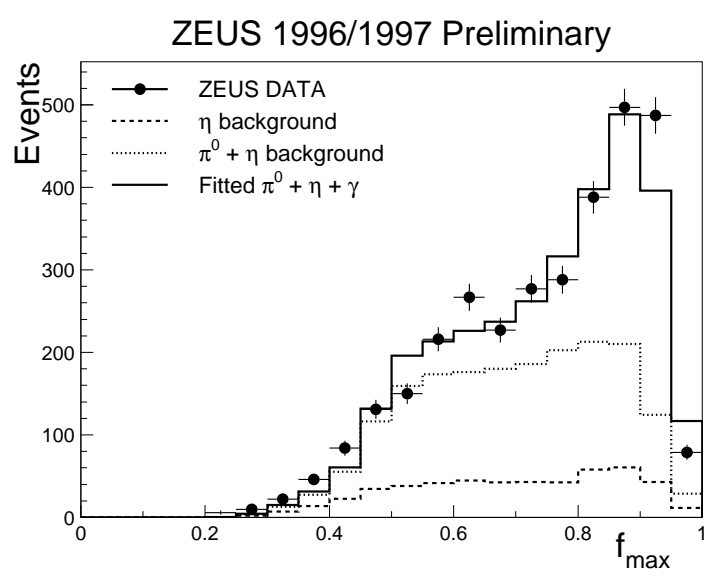

Figure 2: Distribution of $f_{\max }$ for prompt photon candidates in selected events, after cutting on $<\delta Z>$. Also plotted are fitted Monte Carlo curves for photons, $\pi^{0}$ and $\eta$ mesons with similar selection cuts as for the observed photon candidates.

\section{Signal/background separation}

A typical high- $E_{T}$ photon candidate in the BEMC consists of a cluster of 4-5 cells selected by the electron finder. Two shape-dependent quantities were studied in order to distinguish photon, $\pi^{0}$ and $\eta$ signals. These were (i) the mean width $\left\langle\delta Z>\right.$ of the BEMC cluster in $Z$ and (ii) the fraction $f_{\max }$ of the cluster energy found in the most energetic cell in the cluster. $\langle\delta Z\rangle$ is defined as the mean absolute deviation in $Z$ of the cells in the cluster, energy weighted, measured from the energy weighted mean $Z$ value of the cells in the cluster. Its distribution shows two peaks at low $\langle\delta Z\rangle$ which are identified with photons and $\pi^{0}$ mesons, and a tail at higher values. This tail quantified the $\eta$ background; photon candidates in this region were removed.

The remaining candidates consisted of genuine high $E_{T}$ photons and $\pi^{0}$ and remaining $\eta$ mesons. The numbers of candidates with $f_{\max } \geq 0.75$ and $f_{\max }<0.75$ were calculated for the sample of events occurring in each bin of any measured quantity. From these numbers, and the ratios of the corresponding numbers for the $f_{\max }$ distributions of the single particle samples, the number of photon events in the given bin was evaluated. Further details of the background subtraction method are given in [2]. The distribution of $f_{\max }$ for prompt photon candidates in selected events is shown in fig. 2, well fitted to a sum of photon and background distributions.

\section{Results}

We evaluate cross sections for prompt photon production corrected by means of PYTHIA using GRV photon structures [6]. A bin-by-bin factor is applied to the detector-level measurements so as to correct to cross sections in the specified kinematic intervals calculated in terms of the final state hadron system photoproduced in the range $0.16<y^{\text {true }}<0.8$, i.e. $\gamma p$ centre of mass energies in the range $120-270 \mathrm{GeV}$. The virtuality of the incoming photon is restricted to the range $Q^{2}<1 \mathrm{GeV}^{2}$. When a jet was demanded, the hadron-level selections $E_{T}^{j e t}>5 \mathrm{GeV},-1.5<\eta^{j e t}<1.8$ were imposed. The systematic error of $15 \%$ were taken into account and were finally combined in quadrature. The main contributions are from the energy scale on the calorimeter and the background subtraction.

Fig. 3 shows an inclusive cross section $d \sigma / d \eta^{\gamma}$ for prompt photons in the range $5<E_{T}^{\gamma}<10 \mathrm{GeV}$. Reasonable agreement between data and MC is seen at forward values of rapidity, but the data tend to lie above the MC at negative rapidity. The data are also compared with NLO calculations of Gordon(LG), Krawczyk and Zembrzuski(KZ) [3, 7] using the GS and GRV photon structures [6]. The curves are subject to a calculational uncertainty of $5 \%$, and uncertainties in the QCD scale could raise the numbers by up to $\approx 8 \%$. Away from the most forward directions, the LG calculation using GS tend to lie low, while the LG implementation of the GRV photon structure give a reasonable description of the data. KZ calculation has detailed differences from LG including a box diagram contribution for the process $\gamma g \rightarrow \gamma g$ [8].

In fig. 3 inclusive cross sections $d \sigma / d E_{T}^{\gamma}$ for prompt photons in the range $-0.9<\eta^{\gamma}<0.7$ are compared to the theoretical models. All six theoretical models describe the shape of the data well. However the HERWIG predictions is systematically low. The two NLO calculations are in better agreement with the data, and cannot be experimentally distinguished. Similar features can be seen in fig. 4 which shows cross sections for the production of a photon accompanied by a jet in the kinematic range specified above. The $\mathrm{KZ}$ calculation is too 

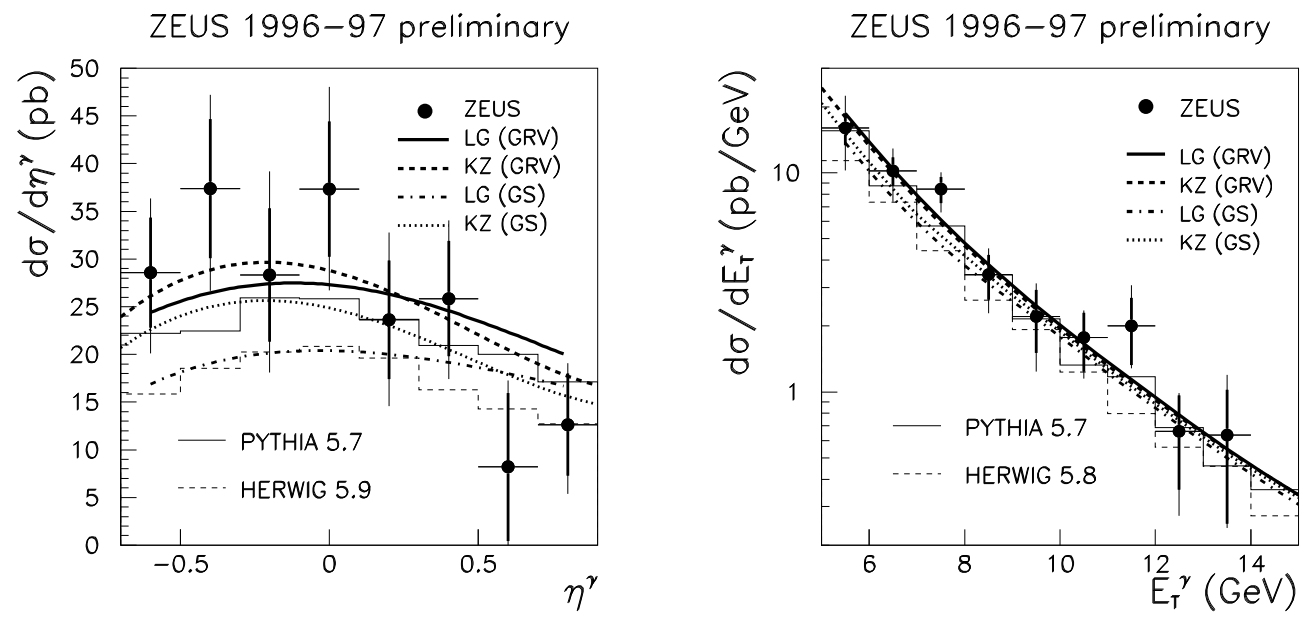

Figure 3: Differential cross sections $d \sigma / d \eta^{\gamma}$ for prompt photons integrated over $5<E_{T}^{\gamma}<10 \mathrm{GeV}, d \sigma / d E_{T}^{\gamma}$ for prompt photons integrated over $-0.7<\eta^{\gamma}<0.9$. Inner (thick) error bars are statistical, outer include systematic added in quadrature. Also plotted are PYTHIA, HERWIG and NLO calculations of LG and KZ with two different photon structures.
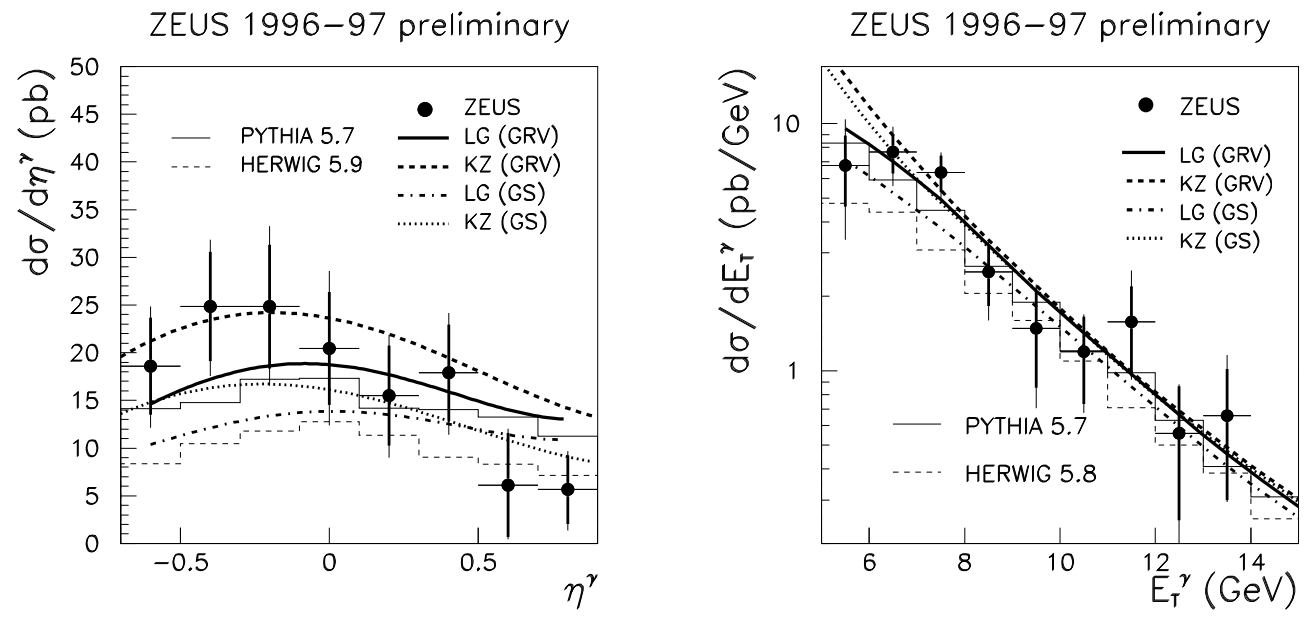

Figure 4: Differential cross sections $d \sigma / d \eta^{\gamma}, d \sigma / d E_{T}^{\gamma}$ for prompt photons with a jet requirement. Also plotted are PYTHIA, HERWIG and NLO calculations of LG and KZ with two different photon structures.

high at low $E_{T}^{\gamma}$, attributable to the lack of a true jet algorithm in this approach [7].

Fig. 4 shows corresponding cross sections for the photon accompanied by at least one jet. The results were corrected to hadron-level jets in the kinematic range $E_{T}^{\text {jet }}>5 \mathrm{GeV},-1.5<\eta^{\text {jet }}<1.8$. In a comparison with NLO calculations from [3, 7] the GS photon structure again provides a less good description of the data overall than that of GRV.

As with the photoproduction of a dijet final state [5], the information from the prompt photon and the measured jet can be used to measure a value of $x_{\gamma}$, the fraction of the incoming photon energy which participates in the hard interaction. A "measured" value of $x_{\gamma}$ at the detector level was evaluated as $x_{\gamma}^{\text {meas }}=\sum(E-$ $\left.p_{Z}\right) / 2 E_{e} y_{J B}$, where the sum is over the jet plus the photon. The resulting distribution is shown in fig. 5 compared with PYTHIA predictions. Reasonable agreement is seen, and a dominant peak near unity indicates clearly the presence of the direct process. Corrected to hadron level, the cross section integrated over $x_{\gamma}>0.8$ is $15.4 \pm 1.6$ (stat) \pm 2.2 (sys) pb. This may be compared with results from Gordon 3, which vary in the range 13.2 to $16.6 \mathrm{pb}$ according to the photon structure taken and the QCD scale (approximately an 8\% effect). Here, the experiment is in good agreement with the range of theoretical predictions but does not discriminate between the quoted models. 


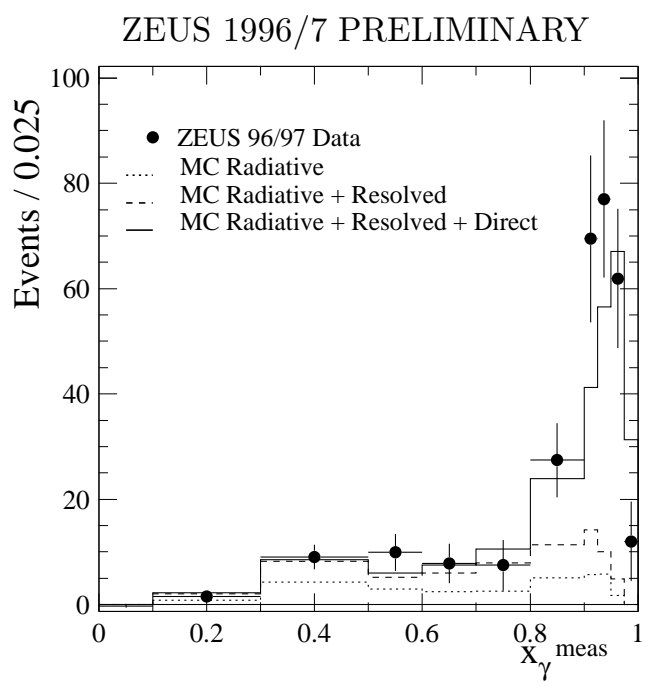

Figure 5: $x_{\gamma}^{\text {meas }}$ associated with the photon+jet final state at the detector level, compared with PYTHIA predictions. Points $=$ data; dotted $=\mathrm{MC}$ radiative; dashed $=\mathrm{MC}$ radiative + resolved; solid line $=\mathrm{MC}$ radiative + resolved + direct.

\section{Conclusions}

The photoproduction of inclusive prompt photons, and prompt photons accompanied by jets, has been measured with the ZEUS detector at HERA using an integrated luminosity of $37 \mathrm{pb}^{-1}$. Cross sections as a function of pseudorapidity and transverse energy have been measured for photon transverse energies in the range $5<E_{T}^{\gamma}<$ $10 \mathrm{GeV}$ and for jet transverse energies in the range $E_{T}^{\text {jet }}>5 \mathrm{GeV}$. The results are compared with partonshower Monte Carlo simulations of prompt photon processes and with NLO QCD calculations incorporating the currently available parameterisations of the photon structure. NLO QCD calculations describe the shape and magnitude of the measurements reasonably well.

\section{Acknowledgments}

We are grateful to L. E. Gordon, Maria Krawczyk and Andrzej Zembrzuski for helpful conversations, and for providing theoretical calculations.

\section{References}

[1] L. E. Gordon and W. Vogelsang, Phys. Rev. D 50 (1994) 1901, Phys. Rev. D 52 (1995) 58

[2] ZEUS Collab., M. Derrick et al., Phys. Lett. B 413 (1997) 201;

Proc. Photon 97, Egmond aan Zee, eds. A. Buijs and F. Erné, World Scientific, Singapore,1998), 150.

[3] L. E. Gordon, Phys. Rev. D 57 (1998) 235

[4] ZEUS Collab., M. Derrick et al., Phys. Lett. B 293 (1992) 465

[5] ZEUS Collab., M. Derrick et al., Phys. Lett. B 348 (1995) 665

[6] L. E. Gordon and J. K. Storrow, Nucl. Phys. B 489 (1997) 405;

M. Glück, E. Reya and A. Vogt, Phys. Rev. D 46 (1992) 1973.

[7] M. Krawczyk and A. Zembrzuski, hep-ph/9810253; priv. comm.

[8] B. L. Combridge, Nucl. Phys. B 174 (1980) 243. 